\title{
Application Analysis of Dry Dust Removal Technology
}

\author{
Dongxing $\mathrm{HUO}^{1, \text { a }}$, Jinglong $\mathrm{LIANG}^{1, \mathrm{~b}}$ and $\mathrm{Yu} \mathrm{YANG}^{1, \mathrm{c}}$ \\ ${ }^{1}$ College of Metallurgy and Energy, North China University of Science and \\ Technology, Tangshan Hebei 063009, China \\ a1158894892@qq.coma, ${ }^{b}{ }^{1 j} @$ ncst.edu.cn, ${ }^{c} 1016737925 @ q q . c o m$
}

Key words: dry dust removal; electrostatic precipitator; the flue gas

Abstract: Introduces the dry process dusting system of converter process flow, equipment composition and function. Practice showed that the dry dust removal system stable running, high efficiency of dust collection, after purification of flue gas dust content is low, operation maintenance cost is low.

\section{Introduction}

Our existing converter gas purification and recovery system using traditional wet dedusting system (OG), low levels of automation control, low recovery rate of per ton steel gas, with an average of $70 \sim 80 \mathrm{~m} 3$, purified gas dust content still amounted to $100 \mathrm{mg} / \mathrm{m} 3$, the gas holder to wet electrostatic precipitator, the recovery system of large energy consumption, energy consumption of per ton steel process for $23.6 \mathrm{~kg}$ of standard coal. And the converter gas dry dust removal system (DDS) per ton of coal gas recovery capacity is $100 \mathrm{~m} 3$ gas containing dust $\mathrm{m} 3$, energy consumption of per ton steel process only $10 \mathrm{~kg}$ standard coal ${ }^{[1]}$. Therefore, the dry dust removal technology of converter flue gas is considered as the future development direction.

\section{Introduction of dry dust removal technology}

For example, Echeng steel 130t converter with top and bottom blowing process, annual output is 140 million $\mathrm{T}$, introduction of Siemens VAI dry dust removal technology, dedusting process from the flue gas into evaporative cooler to gas cooler, mainly composed of evaporative cooler, Yuan Tongjing electric precipitator, axial flow fan, switch station, gas cooler and other equipment. Electrostatic precipitator, evaporative cooler body is the main equipment in the domestic manufacturing; evaporative cooler nozzle, high pressure electrostatic precipitator equipment, axial flow fan and a control system, a switching station key equipment from Siemens VAI company introduction.

Dry dust removal system process flow: about 1500e of the converter gas in the axial flow fan of the pumping effect, through the flue gas cooling system (active smoke cover, fixed smoke cover and vaporization cooling flue), the temperature dropped to $800 \sim 1100 \mathrm{e}$ after entering the evaporative cooler. Evaporative cooler in 10 double medium atomization cooling nozzle of flue gas of cooling, quenching and tempering, coarse dust, the temperature is reduced to $150 \sim 200 \mathrm{e}$, and $30-40 \%$ of dust caught in the evaporative cooler, the formation of coarse grain dust by chain conveyor, double valve plate into the bunker is transported by car. After cooling, coarse dust and quenching and tempering of the flue gas into 4 electric field of a cylindrical electrostatic precipitator, the dust of the flue gas after the electrostatic precipitator to $10 \mathrm{mg} / \mathrm{m} 3$ the following. The fine dust collected in the electrostatic precipitator after fan scraper device, at the bottom of the chain conveyor, spiral transport machine ash and bucket elevator sent to fine ash silo by Sinotrans motor vehicle to 
synthetic material factory, was eventually sintering plant recycling. After the fine dust of the electrostatic precipitator qualified flue gas after the gas cooler cooling to $70 \mathrm{E}$ about to enter the gas tank, unqualified flue gas through the torch dispersing device. The whole system adopts automatic control, which is associated with the control of the converter.

\section{Dry dust removal system structure and function}

Dry dust removal system includes: flue gas cooling system, flue gas purification and recovery system and control system ${ }^{[2]}$.

Converter flue gas cooling system is a waste heat boiler system, which is the cooling system of forced circulation and natural circulation. The working flow of the system is required for soft water by plant network, and sent to a soft water tank, by soft water sent to the deaerator, water deaerator tank part by low pressure forced circulation pump sent to the movable smoke hood inlet pipe, through the movable smoke hood, again through a return pipe return deaerator formed low pressure forced circulation system; part consists of waste heat boiler feed water pump to drum, heat accumulator replenishment. The drum water down pipe sent to the flue, water vaporization flue soda mixture formed by the upward pipe return drum. For the converter vaporization cooling steam get full use and recycling, and make the network from the converter smelting period affecting the steam supply pressure fluctuations, set the accumulator. The heat of steam produced by the boiler automatic control valve into the vapor through network.

The operating condition of the dry dust removal system can be satisfied with the high temperature flue gas containing a large number of $\mathrm{CO}$ after the converter smelting. The flue gas temperature at the inlet of the evaporative cooler is $800 \sim 1100 \mathrm{e}$, and the outlet temperature is about $200 \mathrm{E}$ to reach the condition of the electrostatic precipitator. To this end, the use of 10 pairs of dual channel steam mist nozzle to adjust the optimal water temperature. The water nozzle can be adjusted according to the heat content into the dry gas in the evaporative cooler. Through the steam to make the water atomization into tiny droplets, the water droplets are heated by the flue gas, and the heat of the flue gas is absorbed by the process of vaporization, thus reducing the flue gas temperature. The reduction of the evaporation cooler and the reduction of the gas flow and the wetting of the dust on the inlet of the inlet can be separated from the dust of the coarse particles, and the purpose of the dust removal is achieved. In addition, the evaporative cooler can adjust to improve the gas, that is to reduce the gas temperature while improving its dew point, change the dust than the resistance, is conducive to the separation of dust in the electrostatic precipitator.

The electrostatic precipitator is placed in the outer of the steel making shop, and the dust collector is connected to the outer casing of the dust collector. A high pressure frame is arranged between the dust collector electrode, and the frame is provided with a discharge electrode (cathode) and a high voltage power supply system, which is supported by the insulator. In discharge electrode adjacent to the regional voltage is high strength, as a result of corona discharge voltage, resulting in formation of a negatively charged gas ions, in the discharge electrodes and collecting dust between the electrodes of electric field under with negatively charged gas ions migration to the dust collecting electrode charged with. Which has been part of the gas dust ion ions also negatively charged, free move to the collecting electrode. The fine particles that accumulate in the dust collecting electrode are in the bottom of the electrostatic precipitator through the vibrating system.

DDS is a fan of the axial flow fan, which is a major feature of it. Because of the use of axial flow fan, the smoke in the fan is in the channel, when the electrostatic dust collector in the explosion, can pass through a part of the explosive gas, reducing the explosion. As the dust of the dust in the dust is very low, it is not required to clean the fan impeller. 
And DDS no venturi throat outlet regulating device, micro-differential pressure depends on the wind machine to adjust. Therefore, the flue gas flow rate feedback control system is very necessary. A furnace gas quantity according to blowing oxygen and liquid steel decarburization speed and other parameters to establish a mathematical model; exhaust volume is the DDS in the traffic detection results and flue gas composition measurement results are inputted into the computer. After the incidence of and a gas furnace mathematical model were calculated, through a variable frequency adjustable speed device to change the fan speed, to control the pumping volume with the change of a gas stove, and at the same time control micro-differential pressure, and the content of $\mathrm{CO}$ gas recovery to achieve the best value. Due to the DDS resistance loss of small, mouth micro difference range can be controlled in the range of $0.01 \mathrm{kPa}$; and the resistance loss of the OG, the micro-differential pressure value only can be controlled in range of at.

The dry dust control system is set up with a basic automation, and the automatic system of converter body and steam drum is connected with the network, which is composed of Ethernet optical fiber ring network, and its automation control level is high, which has the function of adaptive control software ${ }^{[3]}$. The dry dust control system is divided into 3 control loops: the temperature control of the evaporative cooler, the flow control of the air blower, and the gas analysis gas composition control.

The temperature of the evaporative cooler is controlled according to the inlet gas temperature and the flow rate of the cooling water to ensure that the flue gas outlet temperature is within the control range. The flow of the flue gas in the cooling and dust removal device is determined by the flow control system. The static pressure in the cooling duct is the main parameter to determine the gas flow rate, and the parameters of the air flow regulation are the oxygen blowing and the flue gas quantity. In the normal operation of the converter, the 1 computer is responsible for handling these 3 parameters and will be used as a parameter to adjust the speed of the fan, such as ore or limestone, or auxiliary operation in the process of steelmaking.

\section{Conclusion}

The dust removal efficiency of the dry dust removal technology of converter gas is high, which can greatly reduce the new water consumption, realize the efficient recycling of the two energy sources, reduce the environmental pollution effectively, and it is an important technical way for the modern iron and steel works to achieve energy saving and emission reduction, development of circular economy.

With the implementation of national energy saving and emission reduction policies, many iron and steel enterprises need to adopt new technology and new technology to achieve energy-saving emission reduction targets. The dry dust removal technology is an effective way to realize the negative energy steelmaking in converter steelmaking.

Dry dust removal system has been put into use so far, running normally, high efficiency of dust removal, the country has created a large economic and social benefits.

\section{Acknowledgements}

This work was financially supported by the National Natural Science Foundation of China (Grant No. 51474093)

\section{Reference}

[1] Xiaobo Wang, Chun Wan. The application of dry dust removal technology in 130t converter [J]. 
Steel Technology, 12-13 2010.In Chinese

[2] Dongli Zhang, Yanli Mao,YuLing Qu. Application of dry dust removal technology for converter gas dry dust [J]. Metallurgical Management, 2010.In Chinese

[3]MASYUKI KAWAMOTO.Recent development of steelmaking process in sumitomo metals

[J].Journal of Iron and Steel Research International, 2011, 18(s2): 28 .In Chinese 